Pacific

Journal of

Mathematics

EXTENDING MAPS OF A CANTOR SET PRODUCT WITH AN ARC TO NEAR HOMEOMORPHISMS OF THE 2-DISK

Michael D. Sanford and Russell B. Walker 


\title{
EXTENDING MAPS OF A CANTOR SET PRODUCT WITH AN ARC TO NEAR HOMEOMORPHISMS OF THE 2-DISK
}

\author{
Michael D. SAnford And Russell B. Walker
}

\begin{abstract}
We prove that a positive entropy map of the product of a Cantor Set and an arc (which covers a homeomorphism) cannot be "embedded" into a near homeomorphism of the 2disk. Thus a theorem of M. Brown cannot be used to embed the induced shift map on the corresponding inverse limit space into a 2-disk homeomorphism.
\end{abstract}

\section{Introduction.}

In 1990, M. Barge and J. Martin [BM90] proved that the shift map on the inverse limit space $([0,1], f)$, for any map $f:[0,1] \rightarrow[0,1]$, can be realized as a global attractor in the plane. In 1960, M. Brown [Bro60] proved that the inverse limit space of any near homeomorphism (Definition 1.2) of a compact metric space is homeomorphic to the original space. M. Barge and J. Martin prove that, for all such $f$, there exists an embedding $h:[0,1] \rightarrow D^{2}$ such that $h \circ f \circ h^{-1}$ can be extended to a near homeomorphism of the 2-disk, $D^{2}$. They then use M. Brown's theorem to extend the induced shift homeomorphism on $h([0,1])$ to a homeomorphism of $D^{2}$. With care in the construction of the near homeomorphism of $D^{2}$, the inverse limit space $\left(h([0,1]), h \circ f \circ h^{-1}\right)$ becomes a global attractor.

The main goal of this paper is to show that analogous techniques for maps, $F: C \times[0,1] \rightarrow C \times[0,1]$, where $C$ is a Cantor set, $F(x, y)=\left(F_{1}(x), F_{2}(x, y)\right)$ is a surjective map with positive topological entropy (Definition 1.4), and $F_{1}$ is a homeomorphism, do not work; no near homeomorphic extension of $h \circ F \circ h^{-1}$ to $D^{2}$ exists for any embedding $h: C \times[0,1] \rightarrow D^{2}$ (Theorem 3.1). In our terminology, such $F$ cannot be "embedded" into any 2-disk homeomorphism (Definition 1.1). In the proof of Theorem 3.1 one first assumes that $h$ is a "tame" embedding (Definition 3.1). But in recent work, R. Walker proves that all embeddings of $C \times[0,1]$ into $D^{2}$ are tame [Wal].

Our study of maps of $C \times[0,1]$ and their embeddings has links to a central problem in the dynamical systems of positive entropy homeomorphisms of compact surfaces.

Does there exist a $C^{1}$ positive entropy 2-disk diffeomorphism without shifts? 
In 1980, A. Katok [Kat80] proved that all $C^{1+\alpha}, \alpha>0$, positive entropy diffeomorphisms of a compact surface, have transverse homoclinic points. So some power of such a diffeomorphism restricts to a shift map of finite type. The next year M. Rees announced a minimal positive entropy homeomorphism of the 2-torus [Ree81]. So her homeomorphism has no periodic orbits thus no shifts. Though not in print, it appears that techniques M. Rees used can be adapted to build a positive entropy 2-disk homeomorphism which has a fixed point and no other periodic orbits. The $C^{1}$ case remains open. In 1993, M. Barge and R. Walker built a chainable continuum which is the inverse limit space of a map of a Cantor comb [BW93]. The map restricted to each "tooth" was a tent map over an adding machine base map. The induced shift homeomorphism has positive entropy but all periodic orbits are period a power of 2 . Thus no shifts are present. All chainable continua can be embedded into the 2-disk [Bin62]. Although their Cantor comb map can be embedded into a near homeomorphism of the 3-ball, it cannot be embedded into a near homeomorphism of the 2-disk. (To prove this M. Barge and $\mathrm{R}$. Walker rely on properties of the adding machine base map.) So their induced shift homeomorphism cannot be used to build a new Rees-type 2disk homeomorphism. By our Theorem 3.1, a much larger class of maps (all positive entropy maps of $C \times[0,1]$ which cover any homeomorphism) has the same drawback.

In Section 2 we show that if $F: C \times[0,1] \rightarrow C \times[0,1]$ is a surjective map such that $F(x, y)=\left(F_{1}(x), F_{2}(x, y)\right), F_{1}$ is a homeomorphism and $F_{2}\left(x_{0}, \cdot\right):[0,1] \rightarrow[0,1]$ is nonmonotone (Definition 1.3) for some $x_{0}$, then there exists no embedding of $F$ into a near homeomorphism (Definition of the 2-disk). We will show this by assuming such a near homeomorphism does exist and then obtaining a contradiction using a result of S. Schwartz [Sch92] (Theorem 1.1) concerning nonmonotone maps.

Unless otherwise specified $X$, and $Y$ are compact metric spaces. And $\pi_{1}$ and $\pi_{2}$ on $X \times Y$ are the first and second coordinate projection maps.

Definition 1.1. A map $f: X \rightarrow X$ can be embedded into the map $F$ : $Y \rightarrow Y$ if there exists a topological embedding $h: X \rightarrow Y$ such that $\left.F\right|_{h(X)}=h \circ f \circ h^{-1}$.

Definition 1.2. A map $f: X \rightarrow Y$ is called a near homeomorphism provided there exists a sequence $\left\{f_{k}: X \rightarrow Y\right\}_{k=1}^{\infty}$ of homeomorphisms which uniformly converge to $f$.

Definition 1.3. A map $f: X \rightarrow Y$ is monotone provided $f^{-1}(V)$ is connected, whenever $V \subset Y$ is connected.

Theorem 1.1 (S. Schwarts [Sch92]). Suppose that $X$ is a locally connected compact metric space. If $f: X \rightarrow X$ is a near homeomorphism then $f$ is monotone. 
As mentioned, in Section 3 we show that if $F: C \times[0,1] \rightarrow C \times[0,1]$ is a surjective map with positive topological entropy (Definition 1.4), which is embedded in the 2-disk, then $F$ cannot be extended to a near homeomorphism of the disk. The proof uses theorems of R. Bowen (Theorem 1.2) [Bow71] and M. Barge (Theorem 1.3) [Bar87].

Definition 1.4 (Topological Entropy). Suppose that $F: X \times Y \rightarrow X \times Y$ is a surjective map and has the form $F(x, y)=\left(F_{1}(x), F_{2}(x, y)\right)$. Fix $x_{0}$ and let $\epsilon>0$. A set $E \subset Y$ is $(n, \epsilon)$-separated by $\left.F\right|_{\pi_{1}^{-1}\left(x_{0}\right)}$ if for all $y_{0}, y_{1} \in E$, $y_{0} \neq y_{1}, d\left(\pi_{2} F^{k}\left(x_{0}, y_{0}\right), \pi_{2} F^{k}\left(x_{0}, y_{1}\right)\right)>\epsilon$ for some $k \in[0, n)$, where $d$ is the $Y$-metric. Since $Y$ is compact and $n<\infty$, card $E<\infty$. Let the maximum number of $(n, \epsilon)$-separated orbits for each $\epsilon$ be

$$
s(n, \epsilon)=\max \left\{\begin{array}{l|l}
\operatorname{card} E & \begin{array}{l}
E \subset Y \text { such that } \\
E \text { is }(n, \epsilon)-\text { separated by }\left.F\right|_{\pi_{1}^{-1}\left(x_{0}\right)}
\end{array}
\end{array}\right\} .
$$

Now, let the growth rate of $s(n, \epsilon)$ (or $\epsilon$-topological entropy) be

$$
h_{\text {top }}\left(\left.F\right|_{\pi_{1}^{-1}\left(x_{0}\right)}, \epsilon\right)=\limsup _{n \rightarrow \infty} \frac{\log s(n, \epsilon)}{n} .
$$

Lastly we let $\epsilon \rightarrow 0$ and define topological entropy for $\left.F\right|_{\pi_{1}^{-1}\left(x_{0}\right)}$.

$$
h_{\text {top }}\left(\left.F\right|_{\pi_{1}^{-1}\left(x_{0}\right)}\right)=\lim _{\epsilon \rightarrow 0} h_{\text {top }}\left(\left.F\right|_{\pi_{1}^{-1}\left(x_{0}\right)}, \epsilon\right) \text {. }
$$

The topological entropy $h_{\text {top }}\left(F_{1}\right)$ of the homeomorphism $F_{1}$ is defined similarly (see [Bow71]).

Theorem 1.2 (R. Bowen [Bow71]). If $F: X \times Y \rightarrow X \times Y$ has the form $F(x, y)=\left(F_{1}(x), F_{2}(x, y)\right)$ then

$$
h_{\text {top }}(F) \leq h_{\text {top }}\left(F_{1}\right)+\sup _{x \in X}\left\{h_{\text {top }}\left(\left.F\right|_{\pi_{1}^{-1}(x)}\right)\right\} \text {. }
$$

If $h_{\mathrm{top}}\left(F_{1}\right)=0$ then $h_{\mathrm{top}}(F)=\sup _{x \in X}\left\{h_{\mathrm{top}}\left(\left.F\right|_{\pi_{1}^{-1}(x)}\right)\right\}$.

Theorem 1.3 (M. Barge [Bar87]). If $F: X \times[0,1] \rightarrow X \times[0,1]$ has the form $F(x, y)=\left(F_{1}(x), F_{2}(x, y)\right), F_{2}(x, \cdot):[0,1] \rightarrow[0,1]$ is monotone for each $x$ and $h_{\mathrm{top}}\left(F_{1}\right)=0$, then $h_{\mathrm{top}}(F)=0$.

\section{Nonmonotone Maps of the Cantor Set Cross the Interval.}

2.1. Preliminaries. Let $C \subset[0,1]$ be a Cantor set. Let $C \times[0,1]$ and $\{\alpha\} \times[0,1] \subset \mathbb{R}^{2}$ for $\alpha \in C$. The goal of this section is to prove Theorem 2.1 to follow. But first some preliminaries.

2.1.0.1. Assume $F: C \times[0,1] \rightarrow C \times[0,1]$ is a surjective map that has the form

$$
F(\alpha, y)=\left(F_{1}(\alpha), F_{2}(\alpha, y)\right)
$$


where $F_{1}: C \rightarrow C$ is a homeomorphism. Furthermore, for a given $\alpha_{0} \in C$, $F_{2}\left(\alpha_{0}, y\right)=t(y)$ where $t:[0,1] \rightarrow[0,1]$ is a continuous nonmonotone map (see Figure 1 for an example). Let $\lambda_{0}=F_{1}\left(\alpha_{0}\right)$.

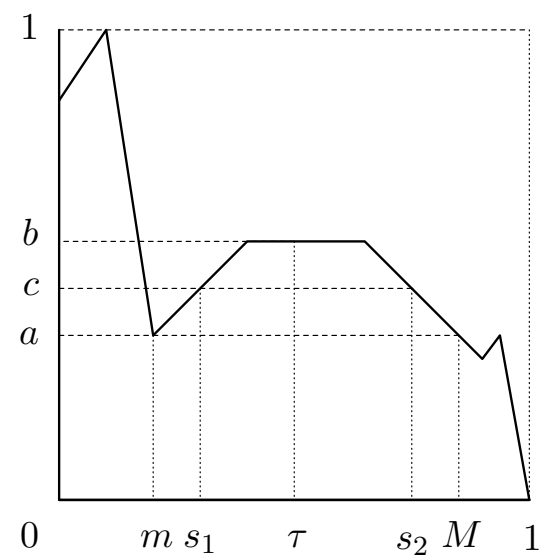

Figure 1. Example of a nonmonotone map.

It will be needed later, that because $t$ is nonmonotone we can find a point that has at least two points in the the pre-image that can be seperated by disjoint epsilon balls. We introduce this idea at this point so that we can use the notation developed here throughout.

2.1.0.2. Since $t$ is nonmonotone and continuous, there is an $a \in(0,1)$ such that $t^{-1}(a)$ is closed and not connected. Thus, there is an interval $(m, M) \subset$ $[0,1] \backslash t^{-1}(a)$ such that $a=t(m)=t(M)$, and $t([m, M])=[a, b]($ or $[b, a])$ for some $b \neq a$. Without loss of generality we will assume that $a<b$. Let $\tau \in t^{-1}(b)$. By the intermediate value theorem, $t([m, M])=[t(m), t(\tau)]$. Now choose $c=\frac{1}{2}(a+b)$. Since $t$ is continuous there are $s_{1} \in(m, \tau)$ and $s_{2} \in(\tau, M)$ such that $c=t\left(s_{1}\right)=t\left(s_{2}\right)$ (see Figure 1).

2.1.0.3. By the continuity of $F$, for any $\epsilon>0$ there is a $\delta_{1}=\delta_{1}(\epsilon)>0$ such that $F(x, y) \in \mathcal{B}_{\epsilon}\left(\lambda_{0}, t(y)\right)$ when $d\left(\alpha_{0}, x\right)<\delta_{1}$ and $y \in[0,1]$. Suppose $K_{1}=K_{1}(\epsilon) \in \mathbb{N}$ is such that $\frac{1}{K_{1}}<\delta_{1}$.

Let $D=\left\{(x, y) \in \mathbb{R}^{2} \mid x^{2}+y^{2} \leq 4\right\}$. Now let $h_{0}: C \times[0,1] \rightarrow D$ be an arbitrary topological embedding. Then there is a homeomorphism $h_{1}: D \rightarrow D$ such that $\left(h_{1} \circ h_{0}\right)\left(\alpha_{0}, y\right)=\left(\alpha_{0}, y\right)$ and $\left(h_{1} \circ h_{0}\right)\left(\lambda_{0}, y\right)=\left(\lambda_{0}, y\right)$ for all $y \in[0,1]$. So $h_{1}$ "straightens out" $h_{0}\left(\alpha_{0} \times[0,1]\right)$ and $h_{0}\left(\lambda_{0} \times[0,1]\right)$ in a strong sense. Notice that $C \times[0,1] \rightarrow D$.

2.1.0.4. By the uniform continuity of $h_{1} \circ h_{0}$, for all $\epsilon>0$ there is a $\delta_{2}=$ $\delta_{2}(\epsilon)>0$ such that for all $y \in[0,1], h_{1} \circ h_{0}(x, y) \in \mathcal{B}_{\epsilon}\left(\alpha_{0}, y\right)$ and $h_{1} \circ$ $h_{0}\left(x^{\prime}, y\right) \in \mathcal{B}_{\epsilon}\left(\lambda_{0}, y\right)$, for all $(x, y) \in \mathcal{B}_{\delta_{2}(\epsilon)}\left(\alpha_{0}, y\right)$ and $\left(x^{\prime}, y\right) \in \mathcal{B}_{\delta_{2}(\epsilon)}\left(\lambda_{0}, y\right)$. Let $K_{2}=K_{2}(\epsilon) \in \mathbb{N}$ be such that $\frac{1}{K_{2}}<\delta_{2}$. 
2.1.0.5. With $a, b$ defined as in [2.1.0.2], let $\hat{d}=\min \{a, 1-b,|a-b|\}$. For $0<\epsilon_{0}<\frac{\hat{d}}{100}$ choose $0<\delta_{0} \leq \min \left\{\delta_{1}\left(\epsilon_{0}\right), \delta_{2}\left(\epsilon_{0}\right), \frac{M-m}{100}\right\}$. So in particular [2.1.0.3] and [2.1.0.4] are satisfied. Note that $t([m, M]) \subset\left[\epsilon_{0}, 1-\epsilon_{0}\right]$. Let $K_{0} \geq \max \left\{K_{1}\left(\epsilon_{0}\right), K_{2}\left(\epsilon_{0}\right)\right\}$ be such that $\frac{1}{K_{0}}<\delta_{0}$. Since $C$ is perfect, there is a sequence $\left\{\alpha_{k}\right\} \subset C$ such that $\alpha_{k} \rightarrow \alpha_{0}$ as $k \rightarrow \infty$, and $\alpha_{k} \times[0,1] \subset$ $\mathcal{N}_{\delta_{0}}\left(\alpha_{0} \times[0,1]\right)$, for all $k>K_{0}$. Let $\lambda_{k}=F_{1}\left(\alpha_{k}\right)$. (Note that $\mathcal{N}_{\delta}(S)$ is a $\delta$-neighborhood of $S$.) It follows that $\lambda_{k} \rightarrow \lambda_{0}$ as $k \rightarrow \infty$ and $\lambda_{k} \times$ $[0,1] \subset \mathcal{N}_{\epsilon_{0}}\left(\lambda_{0} \times[0,1]\right)$ for all $k>K_{0}$. For a possibly larger $K_{0}$, also called $K_{0}$, and $o_{k} \in \mathcal{B}_{\epsilon_{0}}\left(\lambda_{0}, c\right), k>K_{0}$, there exist $q_{1}(k)$ and $q_{2}(k)$ such that $\left\{q_{1}(k), q_{2}(k)\right\} \subset F^{-1}\left(o_{k}\right), q_{1}(k) \in \mathcal{B}_{\delta_{0}}\left(\alpha_{0}, s_{1}\right)$ and $q_{2}(k) \in \mathcal{B}_{\delta_{0}}\left(\alpha_{0}, s_{2}\right)$.

We now state our first theorem.

\subsubsection{Nonmonotone Nonextension Theorem.}

Theorem 2.1. Let $F: C \times[0,1] \rightarrow C \times[0,1]$ be a map of the form $F(\alpha, y)=$ $\left(F_{1}(\alpha), F_{2}(\alpha, y)\right)$ where $F_{1}: C \rightarrow C$ is a homeomorphism. Furthermore, assume $F_{2}\left(\alpha_{0}, \cdot\right):[0,1] \rightarrow[0,1]$ is surjective but not monotone for some $\alpha_{0}$. Then there exists no extension of $h_{0} \circ F \circ h_{0}^{-1}$ to a near homeomorphism of the disk $D$, for any topological embedding $h_{0}: C \times[0,1] \rightarrow D$.

Proof. Assume $h, K_{0}, \epsilon_{0}, \delta_{0},\left\{\alpha_{k}\right\},\left\{\lambda_{k}\right\}, q_{1}(k)$, and $q_{2}(k)$ are defined as in [2.1.0.1-5]. Suppose that $H_{0}: D \rightarrow D$ is a near homeomorphism such that $\left.H_{0}\right|_{h_{0}(C \times[0,1])}=h_{0} \circ F \circ h_{0}^{-1}$. And let $H_{1}: D \rightarrow D$ be given by $H_{1}=h_{1} \circ H_{0} \circ h_{1}^{-1}$. Thus $H_{1}$ is also a near homeomorphism. So the diagram in Figure 2 commutes.

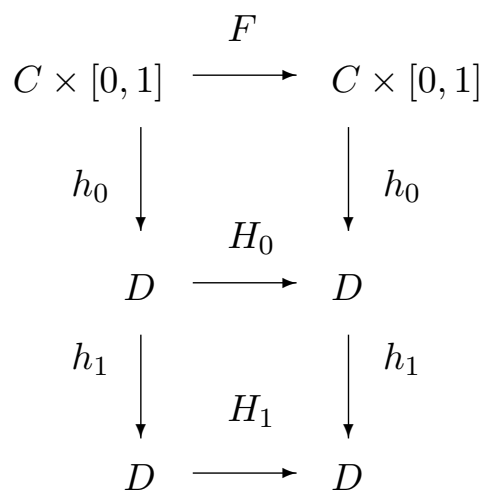

Figure 2. Commuting Diagram.

2.1.1.1. Let $\Lambda(\alpha)=h_{1} \circ h_{0}(\alpha \times[0,1])$ for all $\alpha \in C$. By [2.1.0.3] $h_{1} \circ h_{0}$ is a homeomorphism and if $(\{\alpha\} \times[0,1]) \bigcap(\{\lambda\} \times[0,1])=\emptyset($ when $\alpha \neq \lambda)$, then $\Lambda(\alpha) \cap \Lambda(\lambda)=\emptyset$. Let $\ell_{\beta}$ be the horizontal line $\{y=\beta\}$. And let $\ell_{\beta}^{\alpha}(k)=$ 
$\Lambda\left(\alpha_{k}\right) \bigcap \ell_{\beta}$ and $\ell_{\beta}^{\lambda}(k)=\Lambda\left(\lambda_{k}\right) \bigcap \ell_{\beta}$. Because $h_{1} \circ h_{0}\left(\alpha_{k}, 0\right) \in \mathcal{B}_{\delta_{0}}\left(\alpha_{0}, 0\right)$, $h_{1} \circ h_{0}\left(\alpha_{k}, 1\right) \in \mathcal{B}_{\delta_{0}}\left(\alpha_{0}, 1\right)$, and $\Lambda\left(\alpha_{k}\right)$ is connected, then $\ell_{\beta}^{\alpha}(k) \neq \emptyset$ and all $k \geq K_{0}$ (see Figure 3 and [2.1.0.2]). Similarly $\ell_{\beta}^{\lambda}(k) \neq \emptyset$, for all $\beta \in\left[\epsilon_{0}, 1-\epsilon_{0}\right]$ and $k \geq K_{0}$. Note that if $p \in \ell_{\beta}^{\lambda}(k)$ for given $k \geq K_{0}$ then $p \in \mathcal{B}_{\epsilon_{0}}\left(\lambda_{0}, \beta\right)$.

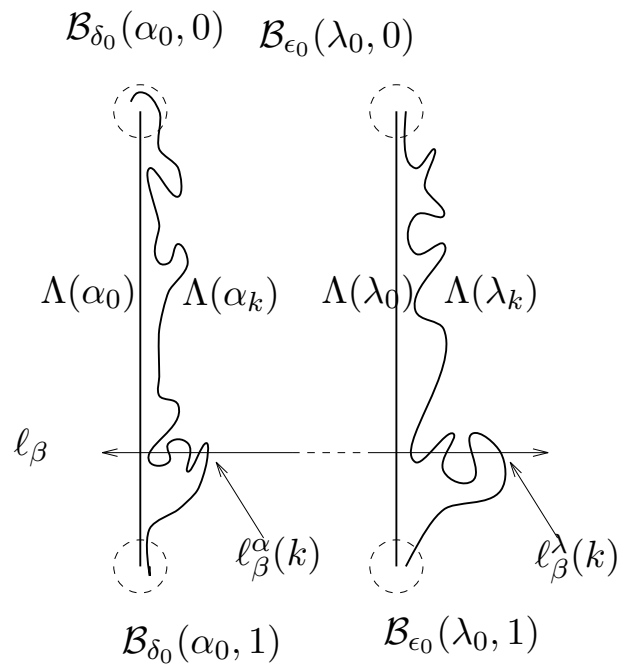

Figure 3. Intersection of $\Lambda\left(\alpha_{k}\right)$ with $\ell_{\beta}$.

Lemma 2.1 follows from the continuity of $h_{1}, h_{0}$, and $\pi_{1}$.

Lemma 2.1. Choose $p_{k} \in \ell_{\beta}^{\alpha}(k)$ for each $k$. Then $\pi_{1} p_{k} \rightarrow \alpha_{0}$ as $k \rightarrow \infty$.

Notice that $\pi_{1}\left(h_{1} \circ h_{0}\right)\left(\alpha_{k}, \frac{1}{2}\right) \neq \alpha_{0}$ for sufficiently large $k$. So either

$$
\operatorname{card}\left\{k \mid \pi_{1}\left(h_{1} \circ h_{0}\left(\alpha_{k}, \frac{1}{2}\right)\right)>\alpha_{0}\right\}=\infty
$$

or

$$
\operatorname{card}\left\{k \mid \pi_{1}\left(h_{1} \circ h_{0}\left(\alpha_{k}, \frac{1}{2}\right)\right)<\alpha_{0}\right\}=\infty .
$$

2.1.1.2. So without loss of generality we may assume there exist distinct $\left\{k_{n}\right\}_{n=1}^{\infty}$ such that $k_{n} \rightarrow \infty$ as $n \rightarrow \infty$, and

$$
\pi_{1}\left(h_{1} \circ h_{0}\left(\alpha_{k_{n}}, \frac{1}{2}\right)\right)>\alpha_{0} .
$$

2.1.1.3. For the sake of simplicity we denote $\alpha_{k_{n}}$ by $\alpha_{n}, \Lambda\left(\alpha_{k_{n}}\right)$ by $\Lambda\left(\alpha_{n}\right)$, $\Lambda\left(\lambda_{k_{n}}\right)$ by $\Lambda\left(\lambda_{n}\right)$ and $\ell_{\beta}^{k_{n}}$ by $\ell_{\beta}^{n}$. 
Lemma 2.2. Let $N_{0}$ be such that $k_{n} \geq K_{0}$ for all $n \geq N_{0}$. Then

$$
\Lambda\left(\alpha_{n}\right) \bigcap\left\{\left(x, \frac{1}{2}\right) \mid x<\alpha_{0}\right\}=\emptyset \text {. }
$$

Proof. Fix $n \geq N_{0}$ and assume there exists

$$
p_{1} \in \Lambda\left(\alpha_{n}\right) \bigcap\left\{\left(x, \frac{1}{2}\right) \mid x<\alpha_{0}\right\},
$$

and let $p_{2}=\left(h_{1} \circ h_{0}\left(\alpha_{k_{n}}, \frac{1}{2}\right)\right)$. By [2.1.1.2] $\pi_{1}\left(p_{2}\right)>0$. Let $A$ be the arc in $\Lambda\left(\alpha_{n}\right)$ with end points $p_{1}$ and $p_{2}$. By [2.1.1.1], $p_{1}, p_{2} \in \mathcal{B}_{\epsilon_{0}}\left(\alpha_{0}, \frac{1}{2}\right)$. So by [2.1.0.5],

$$
d\left(\left(h_{1} \circ h_{0}\right)^{-1}\left(p_{1}\right),\left(h_{1} \circ h_{0}\right)^{-1}\left(p_{2}\right)\right)<\delta_{0} .
$$

Since $\Lambda\left(\alpha_{n}\right) \bigcap \Lambda\left(\alpha_{0}\right)=\emptyset$, then using a Jordan Curve argument, it follows

$$
A \bigcap\left\{\left(\alpha_{0}, y\right) \mid y>1 \text { or } y<0\right\} \neq \emptyset \text {. }
$$

Let $p_{3} \in A \bigcap\{(0, y) \mid y>1$ or $y<0\}$. So $d\left(p_{1}, p_{3}\right)>\frac{1}{2}$. But because $p_{3} \in A$, either

$$
\pi_{2} \circ\left(h_{1} \circ h_{0}\right)^{-1}\left(p_{1}\right)<\pi_{2} \circ\left(h_{1} \circ h_{0}\right)^{-1}\left(p_{3}\right)<\pi_{2} \circ\left(h_{1} \circ h_{0}\right)^{-1}\left(p_{2}\right)
$$

or

$$
\pi_{2} \circ\left(h_{1} \circ h_{0}\right)^{-1}\left(p_{2}\right)<\pi_{2} \circ\left(h_{1} \circ h_{0}\right)^{-1}\left(p_{3}\right)<\pi_{2} \circ\left(h_{1} \circ h_{0}\right)^{-1}\left(p_{1}\right) .
$$

In either case $d\left(\left(h_{1} \circ h_{0}\right)^{-1}\left(p_{1}\right),\left(h_{1} \circ h_{0}\right)^{-1}\left(p_{3}\right)\right)<\delta_{0}$. And so $d\left(p_{1}, p_{3}\right)<\epsilon_{0}$ which is a contradiction.

2.1.1.4. Assume $n \geq N_{0}$. Let $g_{n}:[0,1] \rightarrow \Lambda\left(\alpha_{n}\right)$ be the parameterization of $\Lambda\left(\alpha_{n}\right)$ defined by $g_{n}(\beta)=h_{1} \circ h_{0}\left(\alpha_{n}, \beta\right)$. Using $\tau, m$ from [2.1.0.2] $\Lambda\left(\alpha_{n}\right) \bigcap \ell_{\tau} \neq \emptyset$ and $\Lambda\left(\alpha_{n}\right) \bigcap \ell_{m} \neq \emptyset$ (see Figure 4) so by Lemma [2.2] and the connectivity of $\Lambda\left(\alpha_{n}\right)$ there is a largest $\beta$, call it $\beta_{n}^{-}$, such that $g_{n}\left(\beta_{n}^{-}\right) \in \ell_{m}$. Let $a_{n}=g_{n}\left(\beta_{n}^{-}\right)$(see Figure 4 ). Similarly there is a smallest $\beta$, call it $\beta_{n}^{+}$, such that $g_{n}\left(\beta_{n}^{+}\right) \in \ell_{m}$. Let $b_{n}=g_{n}\left(\beta_{n}^{+}\right)$.

2.1.1.5. If necessary, renumber the $k_{n}$ 's so that if $k_{n}<k_{n+1}$ then $\pi_{1}\left(a_{n}\right)>$ $\pi_{1}\left(a_{n+1}\right)$. It follows by an argument similar to that of Lemma [2.2] that $\pi_{1}\left(b_{n}\right)>\pi_{1}\left(b_{n+1}\right)$. (Because $h_{1} \circ h_{0}$ may have scrambled the $C \times[0,1]$ order in the first coordinate, it may be necessary to relabel the $k_{n}$ 's so that $\Lambda\left(\alpha_{k_{n}}\right)$ to be "between" $\Lambda\left(\alpha_{k_{n-1}}\right)$ and $\Lambda\left(\alpha_{k_{n+1}}\right)$.)

Considering [2.1.1.5] and [2.1.1.2] and to simplify the notation assume

$$
\operatorname{card}\left\{k \mid \pi_{1}\left(h_{1} \circ h_{0}\right)\left(\alpha_{k}, \frac{1}{2}\right)>\alpha_{0}\right\}=\infty
$$

and $\pi_{1}\left(a_{k}\right)>\pi_{1}\left(a_{k+1}\right)$ for all $k$. 


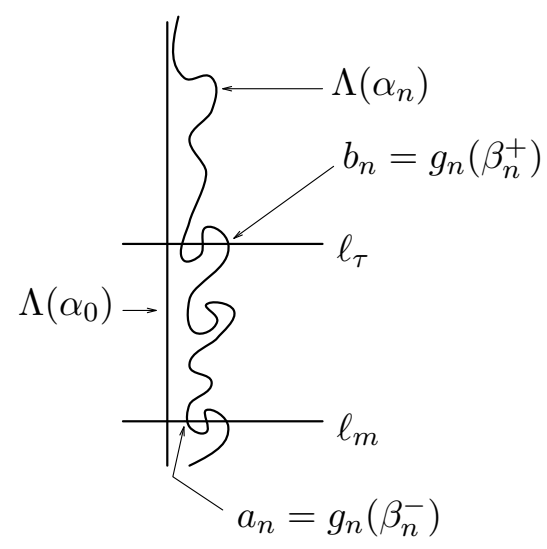

Figure 4. First and Last Intersections.

Using [2.1.1.4], for $k \geq N_{0}$ define the four curves $I(k, m), I(k, \tau), J_{k-1}$, and $J_{k+1}$ in the following manner (see Figure 5$)$. Let $I(k, m)$ be the line segment in $\ell_{m}$ between $a_{k+1}$ and $a_{k-1}$ and $I(k, \tau)$ be the line segment in $\ell_{\tau}$ between $b_{k+1}$ and $b_{k-1}$. Let

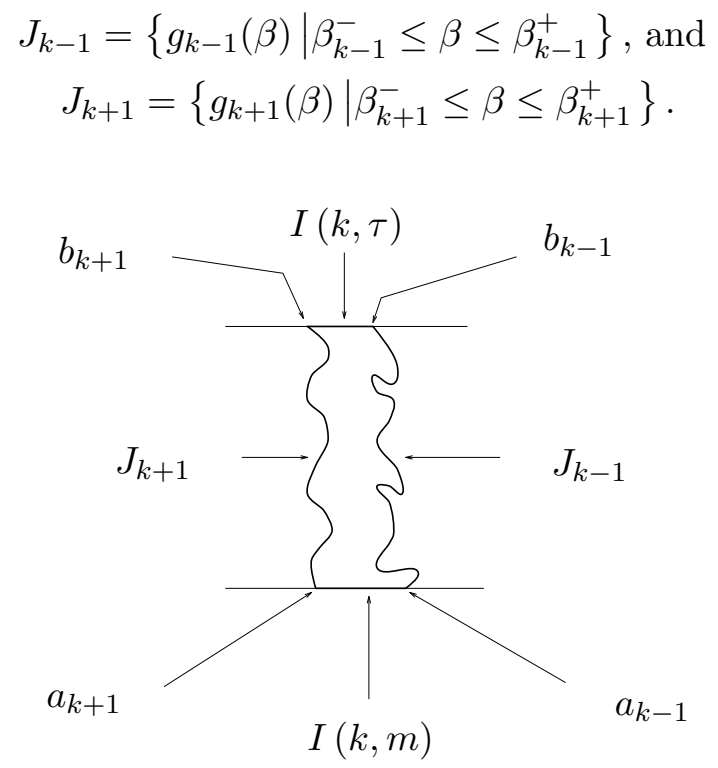

Figure 5. The Boundary of $R_{k}$.

Lemma 2.3. $I(k, \tau) \bigcup J_{k-1} \bigcup I(k, m) \bigcup J_{k+1}$ is a simple closed curve.

Proof. Since $\Lambda\left(\alpha_{k-1}\right) \bigcap \Lambda\left(\alpha_{k+1}\right)=\emptyset$ we have $J_{k-1} \bigcap J_{k+1}=\emptyset$. By [2.1.1.1] $I(k, m) \bigcap I(k, \tau)=\emptyset$. And by [2.1.1.4] we have that

$$
a_{k-1}=J_{k-1} \bigcap I(k, m) \text { and } a_{k+1}=J_{k+1} \bigcap I(k, m)
$$


and

$$
b_{k-1}=J_{k-1} \bigcap I(k, \tau) \text { and } b_{k+1}=J_{k+1} \bigcap I(k, \tau) .
$$

And so the lemma follows.

Let $R_{k}$ be the closed and bounded set with boundary

$$
I(k, m) \bigcup J_{k-1} \bigcup I(k, \tau) \bigcup J_{k+1}
$$

(see Figure 5). Recall from [2.1.0.2] that $s_{1} \in[m, \tau]$ and from [2.1.1.1] that $\ell_{s_{1}}^{\alpha}(k)=\Lambda\left(\alpha_{k}\right) \bigcap \ell_{s_{1}}$ (see Figure 3).

Lemma 2.4. $R_{k} \bigcap \ell_{s_{1}}^{\alpha}(k) \neq \emptyset$.

Proof. Let $\gamma_{k}$ be the arc $\left\{g_{k}(\beta) \mid 0 \leq \beta \leq \beta_{k}^{+}\right\}$. Let $S_{k}=R_{k} \bigcap \pi_{2}^{-1}\left[s_{1}, \tau\right]$ (see Figure 6). So $\partial S_{k} \supset I(k, \tau)$ and by [2.1.1.5] $b_{k} \in I(k, \tau)$. But $b_{k}$ is not an endpoint of $I(k, \tau)$ because the endpoints of $I(k, \tau)$ are $b_{k-1}$ and $b_{k+1}$. And so there is an $\eta>0$ such that if $p \in \mathcal{B}_{\eta}\left(b_{k}\right)$ and $\pi_{2}(p)<\tau$ then $p \in \operatorname{int} S_{k}$. Now, if $q \in \gamma_{k} \backslash\left\{b_{k}\right\}$ then $\pi_{2}(q)<\frac{1}{2}$. And since $\gamma_{k}$ connects $h_{1} \circ h_{0}\left(\alpha_{k}, 0\right)$ to $b_{k}$, we have that $\left(\gamma_{k} \cap \mathcal{B}_{\eta}\left(b_{k}\right)\right) \backslash\left\{b_{k}\right\} \neq \emptyset$. Thus there exists $p_{0} \in \gamma_{k} \cap \mathcal{B}_{\eta}\left(b_{k}\right) \bigcap \operatorname{int} S_{k}$. Let $A_{k} \subset \gamma_{k}$ be the arc with endpoints $p_{0}$ and $h_{1} \circ h_{0}\left(\alpha_{k}, 0\right)$ (see Figure 6).

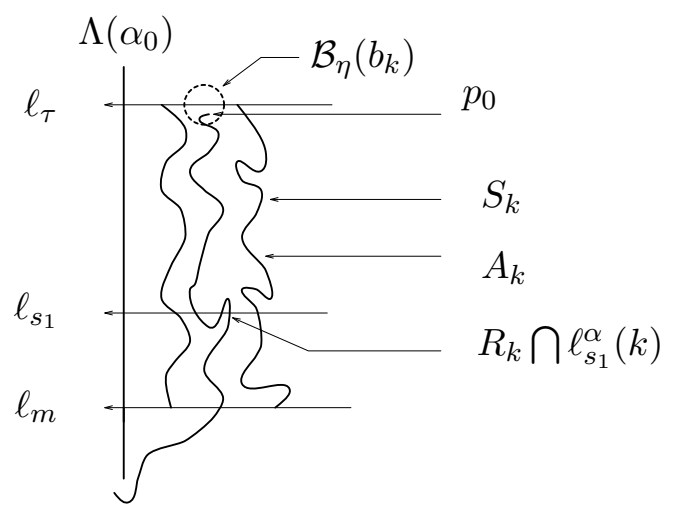

Figure 6. The Arc $A_{k}$.

Because $p_{0} \in \operatorname{int} S_{k}$ and $h_{1} \circ h_{0}\left(\alpha_{k}, 0\right) \notin S_{k}$ then $A_{k} \bigcap \partial S_{k} \neq \emptyset$. Since $A_{k} \bigcap \Lambda\left(\alpha_{k-1}\right)=\emptyset, A_{k} \bigcap \Lambda\left(\alpha_{k+1}\right)=\emptyset, A_{k} \bigcap I(n, \tau)=\emptyset$ and $\ell_{s_{1}} \cap R_{k} \subset \partial S_{k}$, we have that $A_{k} \bigcap \ell_{s_{1}} \cap R_{k} \neq \emptyset$, or $R_{k} \bigcap \ell_{s_{1}}^{\alpha}(k) \neq \emptyset$.

2.1.1.6. Note that since $\ell_{s_{1}}^{\alpha}(k) \bigcap \partial R_{k}=\emptyset$ then $\ell_{s_{1}}^{\alpha}(k) \subset \operatorname{int} R_{k}$.

Lemma 2.5. $\left[\Lambda\left(\alpha_{l}\right) \bigcap H_{1}^{-1}\left(h_{1} \circ h_{0}\left(\lambda_{k}, y\right)\right)\right]=\emptyset$ for $k \neq l$. 
Proof. Suppose that $\rho \in \Lambda\left(\alpha_{l}\right) \bigcap H_{1}^{-1}\left(h_{1} \circ h_{0}\left(\lambda_{k}, y\right)\right)$ for $k \neq l$. Then $H_{1}(\rho)=h_{1} \circ h_{0}\left(\lambda_{k}, y\right)$ But $H_{1} \Lambda\left(\alpha_{l}\right)=\Lambda\left(\lambda_{l}\right)$. Thus $H_{1}(\rho) \in \Lambda\left(\lambda_{l}\right)$ So $h_{1} \circ h_{0}\left(\lambda_{k}, y\right) \in \Lambda\left(\alpha_{l}\right)$ Or $\left(\lambda_{k}, y\right) \in C_{\lambda_{l}}$. Which contradicts, [2.1.1.1] since $k \neq l$.

Proof of Theorem 2.1 continued. By Lemma [2.4] there exists $p_{1}(k) \in$ $R_{k} \cap \ell_{s_{1}}^{\alpha}(k)$ for all $k \geq N_{0}$. By [2.1.0.5] $\left(h_{1} \circ h_{0}\right)^{-1}\left(p_{1}(k)\right) \in \mathcal{B}_{\delta_{0}}\left(\alpha_{0}, s_{1}\right)$. Using [2.1.0.5], let $o_{k}=F \circ\left(h_{1} \circ h_{0}\right)^{-1}\left(p_{1}(k)\right)$. So there exists $\left\{q_{1}(k)\right.$, $\left.q_{2}(k)\right\} \subset F^{-1}\left(o_{k}\right)$ such that $q_{1}(k) \in \mathcal{B}_{\delta_{0}}\left(\alpha_{0}, s_{1}\right)$ and $q_{2}(k) \in \mathcal{B}_{\delta_{0}}\left(\alpha_{0}, s_{2}\right)$. Choose $q_{1}(k)$ so that $p_{1}(k)=h_{1} \circ h_{0}\left(q_{1}(k)\right)$. And let $p_{2}(k)=h_{1} \circ h_{0}\left(q_{2}(k)\right)$ and $r_{k}=h_{1} \circ h_{0}\left(o_{k}\right)$ (see Figure 7). Because $H_{1} \circ h_{1} \circ h_{0}=h_{1} \circ h_{0} \circ F$ then $\left\{p_{1}(k), p_{2}(k)\right\} \in H_{1}^{-1}\left(r_{k}\right)$. By the size of $\delta_{0}$ chosen in [2.1.0.5], $p_{2}(k) \in$ $\mathcal{B}_{\delta_{0}}\left(\alpha_{0}, s_{2}\right) \not \subset R_{k}$.

Recall that $H_{0}$ and $H_{1}$ are near homeomorphisms. Near homeomorphisms are monotone on locally connected compact metric spaces ([Sch92]). Thus pre-images of connected sets under $H_{1}$ are connected. So $H_{1}^{-1}\left(r_{k}\right)$ is a connected set which contains $p_{2}(k) \notin R_{k}$ and by [2.1.1.6] $p_{1}(k) \in \operatorname{int} R_{k}$. Then $H_{1}^{-1}\left(r_{k}\right) \bigcap \partial R_{k} \neq \emptyset$. By Lemma [2.5] either $H_{1}^{-1}\left(r_{k}\right) \bigcap I(k, \tau) \neq \emptyset$ or $H_{1}^{-1}\left(r_{k}\right) \bigcap I(k, m) \neq \emptyset$. So there is an infinite sequence $\left\{\rho_{k_{j}}\right\}$ such that either $\rho_{k_{j}} \in I(k, \tau) \bigcap H_{1}^{-1}\left(r_{k_{j}}\right)$ or $\rho_{k_{j}} \in I(k, m) \bigcap H_{1}^{-1}\left(r_{k_{j}}\right)$ for all $j$ (see Figure 7).

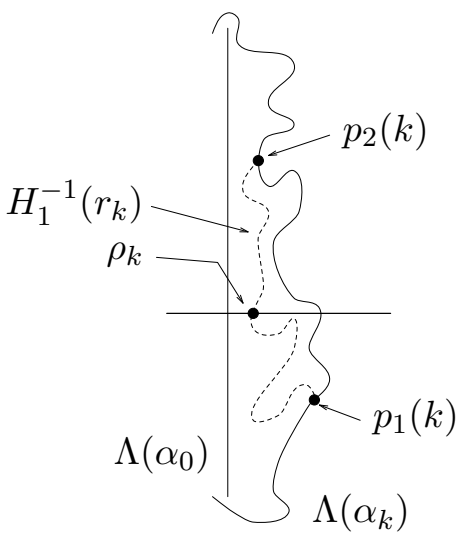

Figure 7. Subsequence and Pre-image.

Now by Lemma [2.1] either $\rho_{k_{j}} \rightarrow h_{1} \circ h_{0}\left(\alpha_{0}, \tau\right)$ or $\rho_{k_{j}} \rightarrow h_{1} \circ h_{0}\left(\alpha_{0}, m\right)$ as $j \rightarrow \infty$. Since $H_{1}$ is continuous for all $j$, either

$$
H_{1} \rho_{k_{j}} \rightarrow H_{1} \circ h_{1} \circ h_{0}\left(\alpha_{0}, \tau\right) \quad \text { or } \quad H_{1} \rho_{k_{j}} \rightarrow H_{1} \circ h_{1} \circ h_{0}\left(\alpha_{0}, m\right) .
$$

Because $H_{1} \circ h_{1} \circ h_{0}=h_{1} \circ h_{0} \circ F$, then either

$$
r_{k_{j}} \rightarrow h_{1} \circ h_{0}\left(\lambda_{0}, t(\tau)\right) \text { or } r_{k_{j}} \rightarrow h_{1} \circ h_{0}\left(\lambda_{0}, t(m)\right) .
$$


Since $h_{1} \circ h_{0}$ is a homeomorphism either

$$
o_{k_{j}} \rightarrow\left(\lambda_{0}, b\right) \quad \text { or } \quad o_{k_{j}} \rightarrow\left(\lambda_{0}, a\right)
$$

which is a contradiction since $\left\{o_{k_{j}}\right\} \subset \mathcal{B}_{\epsilon_{0}}\left(\lambda_{0}, c\right)$.

\section{Positive Entropy Maps of $C \times[0,1]$.}

3.1. Introduction. Let $C \subset \mathbb{R}$ be a Cantor set. In this chapter we use the results of Chapter 2 to prove the following:

Theorem 3.1. Let $F: C \times[0,1] \rightarrow C \times[0,1]$ be a surjective map such that $F(a, y)=\left(F_{1}(a), F_{2}(a, y)\right)$, where $F_{1}: C \rightarrow C$ is a homeomorphism. If $h_{\text {top }}(F)>0$ then there exists no topological embedding $h_{0}: C \times[0,1] \rightarrow D \subset$ $\mathbb{R}^{2}$ such that $h_{0} \circ F \circ h_{0}^{-1}$ extends to a near homeomorphism of the disk $D$.

Recall that $\pi_{1}: C \times[0,1] \rightarrow C$ is the projection map onto the first coordinate. By work of R. Bowen [Bow71] we know that $h_{\text {top }}(F) \leq$ $h_{\text {top }}\left(F_{1}\right)+\sup _{a \in C}\left\{h_{\text {top }}\left(\left.F\right|_{\pi_{1}^{-1}(a)}\right)\right\}$. It has been shown by M. Barge and R. Walker [BW93] that any near homeomorphism that extends $h_{0} \circ F \circ$ $h_{0}^{-1}$ to the disk must preserve a certain local order on the set of fibers $\left\{h_{0}(a \times[0,1]) \mid a \in C\right\}$. But we will show that if $h_{\text {top }}\left(F_{1}\right)>0$ no such local order is preserved. So in fact $h_{\text {top }}\left(F_{1}\right)=0$. Using [Bow71] and a result of M. Barge [Bar87], if $h_{\text {top }}(F)>0$ then for some $a_{0} \in C, F_{2}\left(a_{0}, \cdot\right)$ is a nonmonotone map. Thus by Theorem 2.1, $h_{0} \circ F \circ h_{0}^{-1}$ cannot be extended to a near homeomorphism of the disk.

\subsection{Proof of Theorem 3.1.}

Definition 3.1 (Tame Embedding). $h_{0}: C \times[0,1] \rightarrow D \subset \mathbb{R}$ is a tame embedding provided there is a homeomorphism $h_{1}: D \rightarrow D$ such that for all $a \in C, h_{1} \circ h_{0}(\{a\} \times[0,1])=\left(\left\{a^{\prime}\right\} \times[0,1]\right)$ for some $\left\{a^{\prime}\right\}$. If $h_{0}$ is a tame embedding, using a theorem of E. Moise [Moi77], we may further require that $h_{1}$ has the property: $h_{1} \circ h_{0}(\{a\}, i)=\left(\left\{a^{\prime}\right\}, i\right)$ for all $a$ and $i=0,1$.

For more information concerning tame embeddings see [Rus73] or [Bin54].

3.2.1. Proof of Theorem 3.1. All topological embeddings of $C \times[0,1]$ into $D^{2}$ are tame [Wal]. So it is enough to prove the theorem for all tame embeddings, $h_{0}$.

Let $h_{1}$ be as in Definition 3.1. Denote by $\Lambda$ the set $h_{1} \circ h_{0}(C \times[0,1])$ and by $\Lambda(a)$ the set $h_{1} \circ h_{0}(a \times[0,1])$. Note that $\pi_{1}(\Lambda(a))=a^{\prime}$ for some $a^{\prime} \in \mathbb{R}$. Assume there is a near homeomorphism $H: D \rightarrow D$ such that on $C \times I, h_{1} \circ h_{0} \circ F=H \circ h_{1} \circ h_{0}$. Before continuing with the proof, we stop to define a local ordering on $\{\Lambda(a) \mid a \in C\}$ and prove a lemma. 
3.2.2. Order Definitions and Lemmas. Here we show that $H$ preserves the local order of fibers as defined by M. Barge and R. Walker [BW93], which we will write as $<_{b w}$. And it will follow that $F_{1}: C \rightarrow C$ is a "local order preserving homeomorphism."

Note: Since $h_{0}$ is tame one could use the order on $\{\Lambda(a) \mid a \in C\}$ induced by $\pi_{1}$ in place of $<_{b w}$. That is, $\Lambda(a)<\Lambda(b)$ if $\pi_{1} \Lambda(a)<\pi_{1} \Lambda(b)$. Although $h_{1}$-dependent, this order may be more natural than the $<_{b w}$ order, and is locally equivalent to it. But in order to show that $H$ preserves such a local order on fibers, one must cycle through the definition of $<_{b w}$ in any case.

\section{Barge-Walker order:}

Definition 3.2. For $a, b \in C$ suppose that $\gamma_{-}$and $\gamma_{+}$are arcs in the plane with the properties:

$\gamma_{-}$has endpoints $h_{1} \circ h_{0}(a, 0)$ and $h_{1} \circ h_{0}(b, 0)$, and $\gamma_{-}$is otherwise disjoint from $\Lambda(a) \cup \Lambda(b) ; \gamma_{+}$has endpoints $h_{1} \circ h_{0}(a, 1)$ and $h_{1} \circ h_{0}(b, 1)$ and $\gamma_{+}$is otherwise disjoint from $\Lambda(a) \cup \Lambda(b)$; and

$$
\left(\gamma_{-} \bigcup \gamma_{+}\right) \bigcap\left([0,2] \times\left\{\frac{1}{2}\right\}\right)=\emptyset
$$

Such arcs $\gamma_{-}$and $\gamma_{+}$will be called admissible arcs joining $\Lambda(a)$ and $\Lambda(b)$.

Definition 3.3. Given $a, b \in C, a \neq b$, then $\Lambda(a)<_{b w} \Lambda(b)$ if there are admissible arcs joining $\Lambda(a)$ and $\Lambda(b)$, as above, and the orientation $\gamma_{-} \rightarrow$ $\Lambda(b) \rightarrow \gamma_{+} \rightarrow \Lambda(a)$ is positive (counterclockwise) on the simple closed curve $\gamma_{-} \bigcup \Lambda(b) \bigcup \gamma_{+} \bigcup \Lambda(a)$. (See Figure 8.)

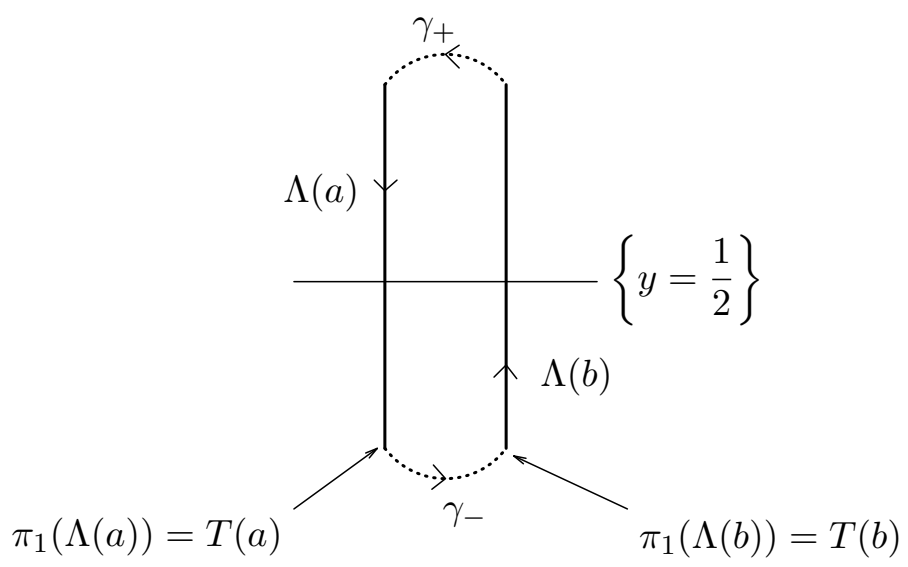

Figure 8. Barge-Walker Ordering on Cantor Fibers.

Definition 3.4. $<_{X}$ is a local ordering on $X$ if for all $x \in X$ there is a $\delta>0$ such that $<_{X}$ is an order relation on $\mathcal{B}_{\delta}(x) .\left(X,<_{X}\right)$ is a locally ordered metric space. 
In [BW93] it is shown that if $a$ and $b$ are sufficiently close, $a \neq b$, then such admissible arcs exist. So either $\Lambda(a)<_{b w} \Lambda(b)$ or $\Lambda(b)<_{b w} \Lambda(a)$. Furthermore $<_{b w}$ is a local ordering on $\Lambda=\{\Lambda(a) \mid a \in C\}$ where we use the metric $d(\Lambda(a), \Lambda(b))=d(a, b)$.

Definition 3.5. Let $a, b \in C$. Then $a<_{C} b$ provided $\Lambda(a)<_{b w} \Lambda(b)$.

It follows from the proceeding remark and that $h_{1} \circ h_{0}$ is uniformly continuous, that $<_{C}$ is a local ordering on $C$.

Definition 3.6. Let $\left(X,<_{X}\right)$ and $\left(Y,<_{Y}\right)$ be locally ordered metric spaces. Let $G:\left(X,<_{X}\right) \rightarrow\left(Y,<_{Y}\right)$ be a homeomorphism. $G$ is a local order preserving homeomorphism, if there is a $\delta>0$ such that if $x_{0}, x_{1} \in X,\left|x_{0}-x_{1}\right|<\delta$, and $x_{0}<_{X} x_{1}$, then $G\left(x_{0}\right)<_{Y} G\left(x_{1}\right)$.

Denote by $[x, y]=\left\{z \in C \mid x \leq_{C} z \leq_{C} y\right\}$. We next show $<_{C}$ on $C$ is $\mathbb{R}$-like in the following sense.

Lemma 3.1. Given $\epsilon>0$ there is a $\delta>0$ such that if $x, y \in C$ and $|x-y|<\delta$, then for all $z \in[x, y],|x-z|<\epsilon$ and $|y-z|<\epsilon$.

Proof. Suppose that $x, y, z \in C$ and $x<_{C} z<_{C} y$. By Definition 3.5 there are admissible arcs $\gamma_{1}^{+}, \gamma_{1}^{-}, \gamma_{2}^{+}$, and $\gamma_{2}^{-}$such that $\Lambda(z) \rightarrow \gamma_{1}^{+} \rightarrow \Lambda(x) \rightarrow \gamma_{1}^{-}$ and $\Lambda(y) \rightarrow \gamma_{2}^{+} \rightarrow \Lambda(z) \rightarrow \gamma_{2}^{-}$have positive orientation.

Sublemma 3.1. For $\epsilon>0$ there is a $\delta_{1}>0$ such that if

$$
\Lambda(z) \bigcap \mathcal{N}_{\delta_{1}}(\Lambda(x)) \neq \emptyset
$$

then $|x-z|<\epsilon$.

Proof. By the continuity of $\left(h_{1} \circ h_{0}\right)^{-1}$, if $\epsilon>0$ there is a $\delta_{1}>0$ such that if $d(p, q)<\delta_{1}$ where $p, q \in \Lambda$ then $d\left(\left(h_{1} \circ h_{0}\right)^{-1}(p),\left(h_{1} \circ h_{0}\right)^{-1}(q)\right)<\epsilon$. So if $\Lambda(z) \bigcap \mathcal{N}_{\delta_{1}}(\Lambda(x)) \neq \emptyset$ there is $p \in \Lambda(x), q \in \Lambda(z)$ such that $d(p, q)<\delta_{1}$. Thus $|x-z|=\left|\pi_{1}\left(\left(h_{1} \circ h_{0}\right)^{-1}(p)\right)-\pi_{1}\left(\left(h_{1} \circ h_{0}\right)^{-1}(q)\right)\right| \leq d\left(\left(h_{1} \circ h_{0}\right)^{-1}(p),\left(h_{1} \circ\right.\right.$ $\left.\left.h_{0}\right)^{-1}(q)\right)<\epsilon$.

Choose $\delta_{1}>0$ smaller so that if

$$
\begin{gathered}
\Lambda(z) \bigcap \mathcal{N}_{\delta_{1}}(\Lambda(x)) \neq \emptyset \text { and } \\
\Lambda(z) \bigcap \mathcal{N}_{\delta_{1}}(\Lambda(y)) \neq \emptyset
\end{gathered}
$$

then $|x-z|<\epsilon$ and $|y-z|<\epsilon$.

By the continuity of $\left(h_{1} \circ h_{0}\right)$ there is $\delta>0$ such that if $|x-y|<\delta$ then $\Lambda(x) \subset \mathcal{N}_{\delta_{1}}(\Lambda(y))$ and $\Lambda(y) \subset \mathcal{N}_{\delta_{1}}(\Lambda(x))$.

Suppose that $\Lambda(z) \bigcap \mathcal{N}_{\delta_{1}}(\Lambda(x)) \bigcap \mathcal{N}_{\delta_{1}}(\Lambda(y))=\emptyset$. So either $\pi_{1} \Lambda(z)<$ $\pi_{1} \Lambda(x)$ or $\pi_{1} \Lambda(y)<\pi_{1} \Lambda(z)$. Thus either $\Lambda(z) \rightarrow \gamma_{1}^{+} \rightarrow \Lambda(x) \rightarrow \gamma_{1}^{-}$has negative orientation or $\Lambda(z) \rightarrow \gamma_{2}^{+} \rightarrow \Lambda(y) \rightarrow \gamma_{2}^{-}$has positive orientation which contradicts $x<_{C} z<_{C} y$. 
Thus $\Lambda(z) \bigcap \mathcal{N}_{\delta_{1}}(\Lambda(x)) \neq \emptyset$ and $\Lambda(z) \bigcap \mathcal{N}_{\delta_{1}}(\Lambda(y)) \neq \emptyset$. So by the choice of $\delta$ then $|x-z|<\epsilon$ and $|y-z|<\epsilon$ as desired.

Lemma 3.2. Let $f:\left(C,<_{C}\right) \rightarrow\left(C,<_{C}\right)$ be a local order preserving homeomorphism. Then there is a $\delta>0$ such that if $|x-y|<\delta$ then $f([x, y])=$ $[f(x), f(y)]$.

Proof. By Definition 3.6 there is an $\epsilon>0$ such that for any $x, y \in C$ if $|x-y|<\epsilon$, and $x<_{C} y$ then $f(x)<_{C} f(y)$. By Lemma 3.1 there is a $\delta>0$ such that if $x<_{C} z<_{C} y$ and $|x-y|<\delta$ then $|x-z|<\epsilon$ and $|y-z|<\epsilon$. Thus $f(x)<_{C} f(z)$ and $f(z)<_{C} f(y)$.

The proof of the following lemma was suggested by M. Barge.

Lemma 3.3. Let $f:(C,<) \rightarrow(C,<)$ be a local order preserving homeomorphism. Then $h_{\mathrm{top}}(f)=0$.

Proof. Recall that $S \subset C$ is an $(n, \epsilon)$-spanning set, for $f$ if for all $x \in C$ there is a $y \in S$ such that $\left|f^{k}(x)-f^{k}(y)\right|<\epsilon$ for all $k=0,1,2, \ldots n-1$. Then $\left(h_{\mathrm{top}}\right)_{\epsilon}(f)=\limsup _{n \rightarrow \infty} \frac{\log \text { card } S(n, \epsilon)}{n}$, and $h_{\mathrm{top}}(f)=\lim _{\epsilon \rightarrow 0}\left(h_{\mathrm{top}}\right)_{\epsilon}(f)$.

Choose $\delta$ as in Lemma 3.2 and suppose that $S \subset C$ is an $(n, \epsilon)$-spanning set where $0<\epsilon \leq \delta$ ( $\delta$ from the lemma). Let $X$ be a finite set of $C$ that is $\epsilon$-dense, let $N=$ card $X$. Before proceeding with the proof of Lemma 3.3 we prove the following sublemma.

Sublemma 3.2. $S \bigcup f^{-n}(X)$ is an $(n+1, \epsilon)$-spanning set.

Proof. Let $x \in C$. Suppose that $y \in S$ is such that $\left|f^{k}(x)-f^{k}(y)\right|<\epsilon$ for $k=0,1,2, \ldots n-1$. There is a $z \in X$ such that either $z \in\left[f^{n}(x), f^{n}(y)\right]$ or $z \in\left[f^{n}(y), f^{n}(x)\right]$, and such that $\left|f^{n}(x)-z\right|<\epsilon$. Then we have that $f^{-n}(z) \in S \bigcup f^{-n}(X)$ and $z$ satisfies $\left|f^{k}(x)-f^{k}(z)\right|<\epsilon$ for $k=0,1,2, \ldots n$ as desired.

Continuing with proof of Lemma 3.3, it follows from Sublemma 3.2 that there exists a constant $K>0$ such that for all $n$, card $S(n . \epsilon) \leq K+n N$. Thus,

$$
\begin{aligned}
h_{\mathrm{top}}(f) & =\lim _{\epsilon \rightarrow 0}\left(h_{\mathrm{top}}\right)_{\epsilon}(f) \\
& =\lim _{\epsilon \rightarrow 0} \limsup _{n \rightarrow \infty} \frac{\log \operatorname{card} S(n, \epsilon)}{n} \\
& =\lim _{\epsilon \rightarrow 0} \limsup _{n \rightarrow \infty} \frac{\log (K+n N)}{n}=0 .
\end{aligned}
$$

Lemma 3.4. Either $H$ or $H^{2}$ locally preserves $<_{b w}$ on $\{\Lambda(a) \mid a \in C\}$. 
Proof. By Theorem 2.1, $\left.H\right|_{\Lambda(c)}$ is monotone for all $c \in C$. Fix $a_{0} \in C$ and assume that $h_{1} \circ h_{0}(\{a\} \times\{i\}) \subset \ell_{i}$ and $H \circ h_{1} \circ h_{0}\left(\left\{a_{0}\right\} \times\{i\}\right) \subset \ell_{i}$ for $i=0$ or 1 . (The other cases are similar.) For all $a \neq a_{0}$ there exists an admissible arc, $\gamma_{a}^{-}$linking $h_{1} \circ h_{0}\left(\left\{a_{0}\right\} \times\{0\}\right)$ to $h_{1} \circ h_{0}(\{a\} \times\{0\})$ and an admissible arc, $\gamma_{a}^{+}$linking $h_{1} \circ h_{0}\left(\left\{a_{0}\right\} \times\{1\}\right)$ to $h_{1} \circ h_{0}(\{a\} \times\{1\})$. Now $H$ is monotone on the simple closed curve $\Gamma=\Lambda\left(a_{0}\right) \bigcup \gamma_{a}^{-} \bigcup \Lambda(a) \bigcup \gamma_{a}^{+}$. Thus $H$ can be approximated by a homeomorphism $H^{\prime}: D \rightarrow D$ such that $H^{\prime} \Lambda\left(a_{0}\right)=H\left(\Lambda\left(a_{0}\right)\right), H^{\prime} \Lambda(a)=H(\Lambda(a)), H \gamma_{a}^{-}=H\left(\gamma_{a}^{-}\right)$, and $H \gamma_{a}^{+}=$ $H\left(\gamma_{a}^{+}\right)$. So the orientation of $H(\Gamma)$ is identical to the orientation of $H^{\prime}(\Gamma)$. For $a$ sufficiently close to $a_{0} H^{\prime}$ (or $\left.\left(H^{\prime}\right)^{2}\right)$ preserves $<_{b w}$ between $\Lambda\left(a_{0}\right)$ and $\Lambda(a)$ [BW93]. Thus $H\left(\right.$ or $\left.(H)^{2}\right)$ does so as well.

Proof of Theorem 3.1 continued. We now complete the proof of Theorem 3.1. First suppose that $F_{1}$ and $F_{1}^{2}$ do not locally preserve $<_{C}$. Then by Definition $3.5 H$ and $H^{2}$ cannot locally preserve $<_{b w}$ on the fibers $\{\Lambda(a) \mid a \in C\}$, contradicting Lemma 3.4.

Next suppose $F_{1}$ locally order preserves $<_{C}$. Then by Lemma 3.3 we have that $h_{\text {top }}\left(F_{1}\right)=0$. And if $F_{1}^{2}$ locally preserves $<_{C}$, then $h_{\text {top }}\left(F_{1}^{2}\right)=0$, thus $h_{\text {top }}\left(F_{1}\right)=0$. So by [Bow71] $h_{\text {top }}(F)=h_{\text {top }}\left(F_{1}\right)+\sup _{a \in C}\left\{h_{\text {top }}\left(\left.F\right|_{\pi_{1}^{-1}(a)}\right)\right\}=$ $\sup _{a \in C}\left\{h_{\text {top }}\left(\left.F\right|_{\pi_{1}^{-1}(a)}\right)\right\}$. But if $h_{\text {top }}(F)>0$ there is an $a_{0} \in C$ such that $h_{\text {top }}\left(\left.F\right|_{\pi_{1}^{-1}\left(a_{0}\right)}\right)>0$. Thus by Theorem 1.3 ([Bar87]) $\left.F_{2}\right|_{a_{0} \times[0,1]}$ is not monotone. So by Theorem 2.1 no such near homeomorphism extension $H$ of $h_{1} \circ h_{0} \circ F \circ\left(h_{1} \circ h_{0}\right)^{-1}$ exists.

\section{References}

[Bar87] M. Barge, The topological entropy of homeomorphisms of Knaster continua, Houston Journal of Mathematics, 13(4) (1987), 465-485.

[BM90] M. Barge and J. Martin, The construction of global attractors, Proc. Am. Math. Soc., 110 (1990), 523-525.

[BW93] M. Barge and R. Walker, Nonwandering structures at the period-doubling in dimensions 2 and 3, Trans. Am. Math. Soc., 337(1) (1993), 259-278.

[Bin54] R.H. Bing, Locally tame sets are tame, Annals of Mathematics, 59 (1954), 145158.

[Bin62] _ Embedding circle like continua in the plane, Canadian J. Math., 14 (1962), 113-128.

[Bow71] R. Bowen, Entropy for group endomorphisms and homogeneous spaces, Trans. Am. Math. Soc., 153 (1971), 401-408.

[Bro60] M. Brown, Some applications of an approximation theorem for inverse limits, Proc. Am. Math. Soc., 11 (1960), 478-483. 
[Kat80] A. Katok, Lyaponov exponents, entropy, and periodic orbits for diffeomorphisms, Publications Mathématique, 51 (1980), 137-173.

[Moi77] E. Moise, Geometric Topology in Dimension 2 and 3, Springer-Verlag, New York, NY, 1977.

[Ree81] M. Rees, A minimal positive entropy homeomorphism of the 2-torus, J. London Math. Soc., 23(2) (1981), 537-550.

[Rus73] T.B. Rushing, Topological Embeddings, Academic Press, 1973.

[Sch92] S. Schwartz, Some Planar Embeddings of Chainable Continua can be Expressed as Inverse Limit Spaces, Ph.D. thesis, Montana State Univ., 1992.

[Wal] R. Walker, Taming the Cantor Fence, Preprint.

Received July 18, 1996 and revised December 16, 1996. This research was supported in part by NSF-OSR grant \#9350546.

262 South Main St.

LODI, NJ 07644

E-mail address: sanfordm@inet.felician.edu

Montana State University

BOZEMAN, MT 59715

E-mail address: walker@math.montana.edu 